\title{
A comparative study of Pap smear findings among HIV positive and negative women at Government Hospital of Thoracic Medicine (GHTM), Tambaram
}

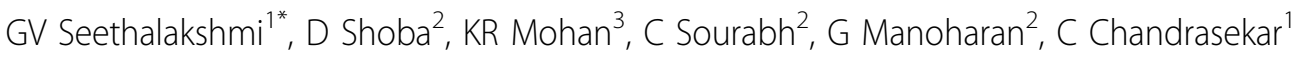 \\ From First International Science Symposium on HIV and Infectious Diseases (HIV SCIENCE 2012) \\ Chennai, India. 20-22 January 2012
}

\section{Background}

Cancer cervix is a major health problem in India, accounting for $26.1-43.8 \%$ of all cancers in Indian women. Dysplasia on Pap smear has been reported in $15-40 \%$ of HIV positive women which is $10-11$ times higher than those observed among HIV negative women. Patients with persistent inflammatory Pap smears harbor high proportion of HPV infection and cervical intraepithelial neoplasia (20.9\%). The purpose of this present study was to compare Pap smear findings in HIV-infected and uninfected women at GHTM and to correlate the Pap smear abnormalities among HIV positive women with their immune status.

\section{Methodology}

All women (18-45 years of age), admitted at GHTM, between July-Sept. 2010, consented were selected and Pap smear was performed. Smears were reported by a pathologist blinded to the HIV status of patients. Results were analyzed by using PASW 18 version.

\section{Results}

Total 300 samples were collected in which 204 were HIV positive and 96 were HIV negative. HIV positive women $(58.8 \%)$ had more Pap smear abnormalities such as cervical dysplasia (3.92\%), inflammatory smear (51.96\%), infection $(2.94 \%)(\mathrm{p}=0.010)$ when compared with HIV negative women (43.75). Among HIV positives 63.93\% had CD4 count less than $250(\mathrm{p}=0.048)$, and
64.3\% had coexisting opportunistic infection $(\mathrm{p}=0.04)$. On speculum examination, cervicitis was present in $63.3 \%$ of HIV positive women with abnormal pap smears $(\mathrm{p}=0.004)$.

\section{Conclusion}

This study showed a high percentage of inflammatory Pap smears (51.96\%) among HIV positives. Hence these patients will require a follow up Pap smear and colposcopy and biopsy if inflammation is persistent to exclude cervical cancer.

\section{Author details}

${ }^{1}$ Government Hospital of Thoracic Medicine (GHTM), Tambaram, Chennai, Tamil Nadu, India. ${ }^{2}$ International Training and Education Centre on Health (ITECH India/AroGyaan), Chennai, Tamil Nadu, India. ${ }^{3}$ Arignar Anna Memorial Cancer Institute and Hospital, Kanchipuram, Tamil Nadu, India.

Published: 4 May 2012

doi:10.1186/1471-2334-12-S1-P35

Cite this article as: Seethalakshmi et al:: A comparative study of Pap smear findings among HIV positive and negative women at Government Hospital of Thoracic Medicine (GHTM), Tambaram. BMC Infectious Diseases 2012 12(Suppl 1):P35.

\footnotetext{
* Correspondence: dermisita@sify.com

${ }^{1}$ Government Hospital of Thoracic Medicine (GHTM), Tambaram, Chennai,

Tamil Nadu, India

Full list of author information is available at the end of the article
} 